\title{
Relato de caso - gestação espontânea em paciente com falência ovariana precoce
}

\section{Case report - spontaneous pregnancy in patient with premature ovarian insufficiency}

\author{
Aline Freire Lucena ${ }^{1,2}$. Michele Coelho Fontenele Sena². Paulo Cruz de Queiroz ${ }^{3}$. \\ 1 Universidade Federal do Ceará (UFC), Fortaleza, Ceará, Brasil. 2 Maternidade Escola Assis Chateaubriand (MEAC), Fortaleza, \\ Ceará, Brasil. 3 Hospital Univeritário Walter Cantídio (HUWC), Fortaleza, Ceará, Brasil.
}

\section{RESUMO}

Objetivos: O trabalho visa mostrar a importância do diagnóstico de falência ovariana precoce (FOP), diminuindo as comorbidades associadas a esta patologia e relatar um caso de gestação espontânea após início de terapia de reposição hormonal (TRH). Metodologia: Revisão de prontuário de paciente atendida no serviço de climatério da Maternidade Escola Assis Chateaubriand. Discussão: Paciente de 21 anos, nuligesta, com desejo de gestar, procura atendimento queixando-se de amenorreia secundária e fogachos intensos. Diagnosticada com FOP após avaliação da anamnese, exame físico e exames laboratoriais. Após 10 meses de tratamento e melhora dos sintomas, engravidou espontaneamente. A FOP incide em $0,1 \%$ das mulheres com menos de 30 anos, e a taxa de gestação espontânea nesses casos varia de 3-10\%. A FOP tem grande impacto na vida da mulher, sendo causa de infertilidade e aumentando a incidência de comorbidades relacionadas à deprivação estrogênica, como osteoporose e risco cardiovascular desfavorável. A maioria das pacientes só engravida após uso de técnicas de reprodução assistida. Conclusão: $\mathrm{O}$ diagnóstico precoce para implementação de tratamento multidisciplinar nas pacientes com FOP é de extrema importância para manutenção da saúde física e mental das pacientes. Incomumente, gestação espontânea pode acontecer, sendo mandatório realizar teste de gravidez se atraso menstrual.

Palavras-chave: Amenorreia. Menopausa precoce. Gravidez. Terapia de reposição hormonal.

\begin{abstract}
Objectives: This article aims to show the importance of diagnosis of early ovarian insufficiency, lowering comorbidities associated with this pathology and to report a case of spontaneous pregnancy after hormonal replacement therapy (HRT). Methodology: Review of a medical record from a patient from Maternidade Escola Assis Chateaubriand. Case: Woman, 21 years, with desire of pregnancy, complaints of amenorrhea and hot flushes. Diagnosed with early ovarian insufficiency after clinical history, physical examination and laboratorial evaluation. After ten months of HRT and improvement of symptoms, spontaneous pregnancy was achieved. Discussion: The disease affects $0,1 \%$ of woman under 30 years and the rate of spontaneous pregnancy in these cases varies in 3-10\%. It is a cause of secondary amenorrhea. The disease has a great impact in women's health, causing infertility and increasing comorbidities related to estrogen withdrawal, such as osteoporosis and high cardiovascular risk. Most patients only reach pregnancy with assisted reproduction techniques. Conclusion: the early diagnosis is very important for the implementation of multidisciplinary treatment for maintaining physical and mental health. Unlikely, pregnancy can occur, being mandatory evaluation for pregnancy.
\end{abstract}

Keywords: Amenorrhea. Menopause, premature. Pregnancy. Hormonal replacement therapy.

Autor correspondente: Aline Freire Lucena, Avenida Rui Barbosa, número 475, Meireles, Fortaleza, Ceará. CEP: 60115-220. Telefone: +55 85 3227-0126. E-mail: aline.flucena@hotmail.com

Conflito de interesses: Não há qualquer conflito de interesses por parte de qualquer um dos autores.

Recebido em: 04 Ago 2018; Revisado em: 08 Dez 2018; Aceito em: 08 Dez 2018. 


\section{INTRODUÇÃO}

A falência ovariana precoce (FOP) é definida por amenorreia por quatro a seis meses associada a hipoestrogenismo e elevação dos níveis séricos de hormônio folículo estimulante (FSH) que se estabelece antes dos 40 anos. ${ }^{1,2} \mathrm{O}$ termo acima não é o mais adequado para descrever a patologia, visto que, em alguns momentos, de forma imprevisível e intermitente, pode ocorrer espontaneamente retorno da função ovariana, sendo mais adequado o termo insuficiência ovariana precoce. ${ }^{3}$

A incidência de FOP é de $1 \%$ para mulheres abaixo de 40 anos, $0,1 \%$ para mulheres com menos de 30 anos e $0,01 \%$ em pacientes com menos de 20 anos. ${ }^{4,5} \mathrm{O}$ diagnóstico precoce é de extrema importância para a saúde feminina, pois as comorbidades associadas são várias, incluindo infertilidade, aumento do risco cardiovascular, osteoporose e desordens neurocognitivas. ${ }^{6}$

Em cerca de $75 \%$ a $90 \%$ dos casos a etiologia é desconhecida, sendo denominada idiopática. ${ }^{7,8}$ As demais causas são doenças autoimunes, principalmente as de tireoide e de suprarrenal (doença de Addison), causas genéticas, principalmente síndrome de Turner ou a síndrome do X frágil, erros inatos do metabolismo (galactosemia), doenças infecciosas, como tuberculose ovariana, varicela, malária, caxumba ou iatrogênicas (quimioterapia e/ou radioterapia). ${ }^{6,7}$

Não há consenso para o diagnóstico de FOP em pacientes jovens, o que pode atrasar o diagnóstico precoce. ${ }^{4} \mathrm{~A}$ apresentação variável, refletindo a flutuação da função ovariana também pode atrasar o diagnóstico. A avaliação deve começar com anamnese e exame físico detalhados, incluindo avaliação de androgenização com escala de Ferriman e índice de massa corporal (IMC). Devem ser solicitados inicialmente beta HCG, FSH, hormônio tireoestimulante (TSH), prolactina e ultrassonografia pélvica. Dosagens dos níveis séricos de androgênios devem ser realizadas nos casos de hiperandrogenismo clínico. ${ }^{6}$

Para o diagnóstico de FOP, as pacientes não podem estar em uso de métodos contraceptivos hormonais durante a investigação. Devem ser excluídas causas de amenorreia secundária e causas iatrogênicas, bem como incluídas dosagens séricas de FSH com valores acima de 25-40UI em duas ocasiões separadas entre quatro a seis semanas. Para avaliação de outras causas mais raras de FOP, podem ser solicitados o painel de autoimunidade, cariótipo e o teste da pré-mutação FMR1, relacionada a síndrome do X frágil. ${ }^{6}$

É de extrema importância a instituição de terapia de reposição hormonal após o diagnóstico, com objetivo de diminuir as comorbidades listadas acima e melhorar a qualidade de vida da paciente ao reduzir os sintomas da síndrome climatérica causada pelo hipoestrogenismo, tais como sintomas vasomotores (fogachos), diminuição da libido e ressecamento vaginal. ${ }^{7}$

O tratamento é baseado na reposição de estrogênio. Se paciente com útero íntegro, deve ser associada reposição de progestágeno, para evitar a hiperplasia endometrial. ${ }^{7} \mathrm{O}$ tratamento visa manter níveis fisiológicos de estrogênio, que pode ser reposto por via transdérmica (primeira escolha), oral ou transvaginal, associada ou não a progestágeno nos últimos dez a catorze dias do ciclo. A reposição hormonal deve ser mantida até a idade média em que ocorre a menopausa, que é em torno de cinquenta anos., ${ }^{4,9}$

\section{RELATO DE CASO}

Paciente nuligesta, de 21 anos, procurou atendimento com queixa, há seis meses, de irregularidade menstrual, com diminuição da duração dos dias de sangramento seguido de fogachos intensos. Apresentou menarca aos 11 anos, com ciclos regulares, que duravam aproximadamente 28 dias, com duração do sangramento de até 7 dias. Negou comorbidades ou cirurgias prévias e não fazia uso de nenhuma medicação. Relatou ter tido na infância infecção por varicela e caxumba. Negou história familiar de FOP e sua mãe teve a menopausa aos 41 anos. Paciente com desejo de gestar, porém, estava sem parceiro no momento. Não foi evidenciada nenhuma alteração no exame físico geral ou ginecológico. Apresentava IMC na faixa de normalidade.

Trazia exames realizados há três meses, cuja única alteração era a dosagem do FSH de 14,9 UI/ml, com dosagem de estradiol de 169,1 pg/ml. Exames mais recentes colhidos cinco dias antes da consulta evidenciavam FSH de $57 \mathrm{UI} /$ $\mathrm{ml}$ e ultrassonografia pélvica que evidenciava útero em anteversoflexão, com volume de $29 \mathrm{~cm}^{3}$, volume com contorno e ecotextura normais, endométrio homogêneo de espessura normal medindo $20 \mathrm{~mm}$, ovário direito medindo $1,5 \mathrm{~cm}^{3}$, e ovário esquerdo $3,4 \mathrm{~cm}^{3}$. Paciente havia sido investigada para tireoidopatias, diabetes e autoimunidade. Novos exames, colhidos após a consulta, mostraram FSH de 91,9 UI/ml e estradiol de $48 \mathrm{pg} / \mathrm{ml}$. Com o quadro clínico acima, foi dado o diagnóstico de hipogonadismo hipergonadotrófico, no caso, falência ovariana precoce, sendo iniciada reposição hormonal com estradiol $1 \mathrm{mg}+$ norestiterona $0,5 \mathrm{mg}$ em dose diária.

Após dois meses de reposição hormonal, paciente relatou melhora do quadro de fogachos. Trouxe novos exames realizados após seis meses do início do tratamento que mostraram dosagem do FSH de 0,6 UI/ml e demais exames dentro da normalidade. Prosseguiu seguimento ambulatorial e, após sete meses de tratamento, iniciou quadro de náuseas matinais. Realizou beta HCG, que teve resultado positivo e ultrassonografia que evidenciou saco gestacional com embrião presente medindo em seu comprimento cabeça nádega $(\mathrm{CCN})$ $13,5 \mathrm{~mm}$, batimento cardiofetal de 164 batimentos por minuto, datando idade gestacional de sete semanas e cinco dias.

Paciente não teve intercorrências no pré-natal. Gestação foi resolvida com 39 semanas e seis dias, sendo indicada via abdominal por desproporção cefalopélvica. Recém-nascida com 3920 g, APGAR 9 no primeiro e quinto minuto.

\section{DISCUSSÃO}

A falência ovariana precoce leva a hipoestrogenismo, que pode ser resolvido com a TRH. O mesmo não pode ser dito em relação 
à função reprodutiva. ${ }^{2}$ No entanto, mulheres com FOP não necessariamente são estéreis, tendo uma chance entre 3-10\% de ovular espontaneamente sem uso de terapias de reprodução se apresentarem cariótipo normal $(46, \mathrm{XX}){ }^{1,9}$ Não há parâmetro clínico ou laboratorial capaz de predizer ovulação nesses casos. ${ }^{3}$

Não há evidência conclusiva que aponte o melhor tratamento para pacientes com FOP que desejam engravidar usando os próprios gametas, entretanto, é importante que os níveis de gonadotrofina (FSH) estejam próximos ao nível da normalidade antes de iniciar qualquer técnica de reprodução assistida. ${ }^{10}$ Até o presente, há pouca informação disponível acerca das características reprodutivas das pacientes portadora de FOP.

Daan et al realizaram um estudo em 2005 com um total de 479 mulheres com FOP, incluindo mulheres com e sem gestação anterior ao diagnóstico. Do total, oito engravidaram espontaneamente. A idade média para gestação foi de trinta anos. Os resultados do estudo apontaram que mulheres com gestação prévia evoluíram mais tardiamente com disfunção ovariana e apresentaram níveis de FSH mais baixos, quando comparadas com pacientes nuligestas. ${ }^{5}$

Maruyama et al reportaram em 2013 quatro casos de FOP que foram acompanhadas com técnicas de reprodução assistida. Todas evoluíram para gestação com embrião próprio em espaço variado de tempo e com uso de diferentes técnicas. Uma das pacientes realizou acompanhamento de crescimento folicular via ultrassonografia por seis anos, apresentando apenas oito ciclos ovulatórios, mostrando a baixa incidência de ovulação espontânea. ${ }^{11}$

A fertilidade é um ponto de controvérsia nas pacientes com FOP. Para aquelas que se mantêm inférteis apesar da reposição hormonal, opções alternativas para gestação seriam receber

\section{REFERÊNCIAS}

1. Asbagh FA, Ebrahimi M. A case report of spontaneous pregnancy during hormonal replacement therapy for premature ovarian failure. Iran J Reprod Med. 2011;9(1):47-9.

2. Egbe TO, Wafo CY, Bollo BB, Pany C, Onomo MJ, Sandjon G. Successful pregnancy with donor eggs in-vitro fertilization after premature ovarian insufficiency in a tertiary jospital in a low-income setting: A case report. Fertil Res Pract. 2016;2:2-12.

3. Bidet M, Bachelot A, Bissauge E, Golmard JL, Gricourt S, Dulon $\mathrm{J}$, et al. Resumption of ovarian function and pregnancies in 358 patients with premature ovarian failure. J Clin Endocrinol Metab. 2011;96(12):2864-3872.

4. Moreira AM, Spritzer PM. Primary ovarian insufficiency: different approaches in three cases and a review of literature. Endocrinol Diabetes Metab Case Rep. 2016;2016:1-6.

5. Daan NM, Hoek A, Corpeleijn E, Eijkemans MJ, Broekmans FJ, Fauser BC, et al. Reproductive characteristics of women diagnosed with premature ovarian insufficiency. Reproductive Biomedicine Online. 2016;32(2):225-32.

\section{Como citar:}

LucenaAF, Sena MC, Queiroz PC. Relato de caso - gestação espontânea em paciente com falência ovariana precoce. Rev Med UFC. 2019 jul-set;59(3):74-76. oócitos ou embriões doados e criopreservação de tecido ovariano (nas pacientes que vão se submeter a quimioterápicos ou radioterápicos). ${ }^{5,9}$ Apesar de haver teorias sobre o aumento da fertilidade com o uso de TRH, investigações não demonstraram evidência clínica do benefício da terapêutica para essa finalidade. ${ }^{9}$

Existem estudos que avaliaram a função ovariana e reprodutiva de pacientes com FOP, porém, ainda não há consenso na literatura sobre qual a melhor técnica para melhorar as taxas de fertilidade com gametas próprios nessas pacientes. ${ }^{10}$ Foram avaliados possíveis critérios preditores positivos na recuperação da fertilidade, tais como níveis baixos de FSH no momento do diagnóstico, ausência de marcadores de autoimunidade e amenorreia secundária. Porém, esses marcadores ainda necessitam de estudos maiores para validação. ${ }^{3}$

Para muitas pacientes com FOP, a questão da infertilidade pode ser a mais devastadora. É de extrema importância que essas pacientes sejam acompanhadas por equipe multidisciplinar com educadores físicos, psicólogos e médicos, que forneça o acompanhamento adequado para melhorar a qualidade de vida das pacientes. ${ }^{6}$

\section{CONCLUSÃO}

O diagnóstico precoce para implementação de tratamento multidisciplinar nas pacientes com FOP é de extrema importância para manutenção da saúde física e mental das pacientes, principalmente a avaliação de osteoporose, aumento do risco cardiovascular e infertilidade.

Diferentes técnicas de reprodução assistida podem ser utilizadas com sucesso. Incomumente, gestação espontânea pode acontecer, sendo mandatório orientação sobre métodos contraceptivos se a paciente não desejar gestar e realizar teste de gravidez se atraso menstrual.

6. Nguyen HH, Milat F, Vincent A. Premature ovarian insufficiency in general practice: Meeting the needs of women. Aust Fam Physician. 2017;46(6):360-6.

7. Assumpção CR. Falência ovariana precoce. Arq Bras Endocrinol Metab. 2014;58(2):132-43.

8. Hernandez-Angeles C, Castelo-Branco C. Early menopause: a hazard to a woman's health. Indian J Med Res. 2016;143(4):420-7.

9. Sullivan SD, Sarrel PM, Nelson LM. Hormone replacement therapy in young women with primary ovarian insufficiency and early menopause. Fertil Steril. 2016;106(7):1588-99.

10. Ben-Nagi J, Panay N. Premature ovarian insufficiency: how to improve reproductive outcome? Climateric. 2014;17(3):242-6. Yoshimura Y. Achievement of pregnancies in women with primary ovarian insufficiency using close monitoring of follicle development: case reports. Endocrine Journal. 2013;60(6):791-7.
11. Maruyama T, Miyazaki K, Uchida H, Uchida S, Masuda H, 